\title{
Evaluation Of Clinical, Laboratory And Radiological Findings In Pediatric Covid-19 Cases And Their Relation With RT-PCR Result
}

\author{
Muhammet Asena ${ }^{1}$, ilyas yolbaş², and murat Kanğın ${ }^{3}$ \\ ${ }^{1}$ Affiliation not available \\ ${ }^{2}$ Dicle Universitesi \\ ${ }^{3}$ Saglik Bilimleri Universitesi
}

February 5, 2021

\begin{abstract}
Background: The pandemic of the new coronavirus disease (COVID-19) continues to be a major health problem globally. In this study, clinical findings, radiological findings, laboratory findings and polymerase chain reaction (PCR) test results were evaluated according to age groups in pediatric patients with COVID-19. Methods: In this study, PCR (+) and PCR (-) 278 cases diagnosed with COVID-19 between March 15, 2020 and September 30, 2020 were assessed considering clinical symptoms, radiological and laboratory findings and RT-PCR test results. Results: $43.9 \%$ of the cases consisted of RT-PCR (+) and $56.1 \%$ of RT-PCR (-) cases. Fever was observed at the rate of $64.7 \%$, cough $53.2 \%$, respiratory distress $12.2 \%$, myalgia $24.5 \%$, diarrhea $12.9 \%$, chest X-ray findings $48.2 \%$ and computed tomography findings $43.6 \%$. Diarrhea and cough, alanine aminotransferase, aspartate aminotransferase, leukocyte and lymphocyte elevation were determined as significantly higher in younger age group cases, while high myalgia and neutrophilia was observed in older age group children $(\mathrm{p}<0.05)$. Fever, high CRP, leukocytosis frequency, high neutrophil, were significantly higher in $\operatorname{PCR}(-)$ cases and the frequency of respiratory distress, high lymphocyte and chest computed tomography findings in $\mathrm{PCR}(+)$ cases $(\mathrm{p}<0.05)$. Conclusion: COVID-19 infection may indicate different nonspecific clinical, laboratory and radiological findings in children according to both adults and pediatric age groups. In addition, the results of the PCR test may give erroneous results in cases due to conditions such as fever, respiratory distress, high CRP, leukocytosis, high neutrophil and CT finding. Keywords: pediatric, COVID-19, RT-PCR
\end{abstract}

\section{Evaluation Of Clinical, Laboratory And Radiological Findings In Pediatric Covid-19 Cases And Their Relation With RT-PCR Result}

Background: The pandemic of the new coronavirus disease (COVID-19) continues to be a major health problem globally. In this study, clinical findings, radiological findings, laboratory findings and polymerase chain reaction (PCR) test results were evaluated according to age groups in pediatric patients with COVID19.

Methods: In this study, PCR (+) and PCR (-) 278 cases diagnosed with COVID-19 between March 15, 2020 and September 30, 2020 were assessed considering clinical symptoms, radiological and laboratory findings and RT-PCR test results.

Results: $43.9 \%$ of the cases consisted of RT-PCR (+) and $56.1 \%$ of RT-PCR (-) cases. Fever was observed at the rate of $64.7 \%$, cough $53.2 \%$, respiratory distress $12.2 \%$, myalgia $24.5 \%$, diarrhea $12.9 \%$, chest X-ray findings $48.2 \%$ and computed tomography findings $43.6 \%$. Diarrhea and cough, alanine aminotransferase, aspartate aminotransferase, leukocyte and lymphocyte elevation were determined as significantly higher in younger age group cases, while high myalgia and neutrophilia was observed in older age group children 
( $\mathrm{p}<0.05)$. Fever, high CRP, leukocytosis frequency, high neutrophil, were significantly higher in PCR(-) cases and the frequency of respiratory distress, high lymphocyte and chest computed tomography findings in $\operatorname{PCR}(+)$ cases $(\mathrm{p}<0.05)$.

Conclusion: COVID-19 infection may indicate different nonspecific clinical, laboratory and radiological findings in children according to both adults and pediatric age groups. In addition, the results of the PCR test may give erroneous results in cases due to conditions such as fever, respiratory distress, high CRP, leukocytosis, high neutrophil and CT finding.

Keywords: pediatric, COVID-19, RT-PCR

\section{INTRODUCTION}

The outbreak of the new coronavirus disease (COVID-19) continues to be a major global health problem that emerged in Wuhan Province, China in late December, turned into a pandemic within a few months and caused many deaths despite many worldwide measures $(1,2)$ Although COVID-19 infection usually presents with symptoms such as fever, cough, respiratory distress, myalgia, and diarrhea in children and adults, it mostly progresses less in children compared to adults, and radiological and laboratory findings may be different in children and adults (3,4). Early diagnosis of COVID-19 infection in children is significant not only for the health of children, but also for preventing the risk of transmission to adults. Although many diagnostic tests are being used for the diagnosis of COVID-19 infection throughout the world, RT-PCR test is accepted as the most reliable laboratory diagnosis method, but the average accuracy of the PCR test is around 40-60\% depending on many factors (5). Therefore, clinical findings, radiological findings and laboratory tests have an important place in the diagnosis and follow-up of COVID-19, especially in pediatric patients $(6,7)$. In this study, clinical findings, radiological findings, laboratory findings and polymerase chain reaction $(\mathrm{PCR})$ test results were evaluated according to age groups in pediatric patients who were followed up with the diagnosis of COVID-19 with PCR (+) or PCR (-).

\section{MATERIAL METHOD}

278 pediatric patients were included in this study, whose files were accessed and treated with the diagnosis of COVID-19 at the Saglik Bilimleri University Diyarbakir Gazi Yasargil Training and Research Hospital COVID-19 clinic between 15 March 2020 and 30 September 2020, were included. The study was approved by the Saglik Bilimleri University Diyarbakir Gazi Yasargil Training and Research Hospital Ethics Committee $(2018 / 20)$. The patients were divided into 2 groups. Patients diagnosed with COVID-19 with PCR $(+)$ and patients who had close contact with COVID-19 patients despite having PCR (-) and who were diagnosed with COVID-19 according to the diagnostic criteria of the World Health Organization with the help of clinical laboratory and radiological findings were included. Patients who had PCR (-) and did not have close contact with COVID-19 patients or whose clinical laboratory and radiological findings did not fully support the diagnosis of COVID-19 were not included in the study. COVID 19 virus infection was diagnosed using nasal or nasopharyngeal swab samples taken in line with national recommendations by trained medical practitioners, and real-time reverse transcriptase polymerase chain reaction tests recommended by WHO (8).

\section{Statistical Analysis}

Statistical Package for Social Sciences (SPSS) for Windows v17.0.0 program was used for the statistical analysis of the data obtained from the patients participated to study. While assessing the obtained data, besides descriptive statistical methods (mean, standard deviation), Student t test was used for comparing normally distributed parameters between groups in comparison of quantitative data, and MannWhitneyU test was used for comparing non-normally distributed parameters between groups. Comparison of percentages between groups was performed through using the Chi-square test.

\section{FINDINGS}

Total of 278 patients, including $122(43.9 \%)$ PCR (+) cases and $156(56.1 \%)$ PCR (-) cases have been assessed. While PCR (-) cases consisted of $86(55.1 \%)$ girls and $70(44.9 \%)$ boys, $60(49.2 \%)$ of the PCR 
$(+)$ cases were girls and $62(50.8 \%)$ were boys $(\mathrm{p}=0.486)$. When the distribution by age groups was taken, the patients included in the study are as follows: $13 \%$ (36) $0-1$ years, $15.2 \%$ (42) 2-3 years, $13 \%$ (36) 4-6 years, $20.3 \%$ (56) 7-11 years and \%38,4 (106) 11-18 years. There was a positive family history in $114(93.4 \%)$ of RT-PCR $(+)$ cases. While $47.4 \%$ (74) of the PCR (-) cases were followed up by hospitalization, $49.2 \%$ $(60)$ of the PCR $(+)$ cases were followed up by hospitalization. Mortality was not observed in any cases. There was also a positive family history in $114(93.4 \%)$ of RT-PCR $(+)$ cases. Diarrhea and Cough, high alanine aminotransferase (ALT), high aspartate aminotransferase (AST), high White Blood Cell (WBC), high lymphocyte were determined statistically significantly higher in younger children, and myalgia and neutrophilia were found to be significantly higher in older children (Table 1). While $47.4 \%$ (74) of the PCR (-) cases were followed by hospitalization, $49.2 \%(60)$ of the PCR (+) cases were

While in PCR (-) cases, the frequency of fever, high CRP, high leukocytosis, neutrophil and CT findings were determined as statistically significantly higher than PCR $(+)$ cases, the frequency of respiratory distress, high lymphocyte and CT findings frequency were determined as statistically significantly higher in PCR $(+)$ cases (Table 2).

\section{DISCUSSION}

Children of all ages, regardless of gender, can be infected with COVID-19 infection, although more cases have been reported in younger children and infants (9). Furthermore, the mean age of infection in children up to age of 15 was determined as 6.7 (9). In their study, Lara $\mathrm{S}$ et al found the rate of boys as $52 \%$ and the median age as 13 (4.2-16.6) years (10). Again, in a recent study, boys 13 (65\%), Girl 7 (35\%), Age <1 month 3 (15\%), 1 month-1 year 6 (30\%), 1-3y 5 (25\%) (11). In this study, the rate of boys in PCR (-) covid-19 cases was found as $44.9 \%$, while the rate of boys in PCR (+) cases was determined as $50.8 \%$. addition, the age distribution was 13\% 0-1 years, $15.2 \%$ 2-3 years, $134-6$ years, $20 \%, 3-10$ years and $38.4 \% 11-18$ years. In the light of the data discussed above, it is possible to say that there is no difference in terms of age or gender in COVID-19 cases (9). Currently, it is difficult to define the clinical characteristics of children with COVID-19 infection since there are small number of scientific clinical studies conducted on children (12). In a study conducted in China, the rate of asymptomatic COVID-19 infection in children was reported as $13 \%$ (13). In a previous study conducted in different countries, while the most common clinical symptoms in children with covid-19 were determined as Cough (\%21-85.1), Fever (\%26-59.2), Shortness of breath (\%2.6759.2), Diarrhea 1 (\%3.7-7.6), jess frequently sore throat, sneezing, fatigue and vomiting have been reported $(3,4,11,14,16)$. Also it has been reported that children may have more upper respiratory symptoms than lower respiratory symptoms and recover within 1-2 weeks $(9,17)$. In this study, the most common clinical symptoms in children were determined as fever $(64.7 \%)$, cough $(53.2 \%)$, respiratory distress $(12.2 \%)$, myalgia $(24.5 \%)$ and diarrhea (12.9\%). In addition, diarrhea and cough symptoms were more common in younger age group children, while myalgia was significantly more common in older age group children. It can be concluded that the use of these clinical findings in the diagnosis of COVID-19 is limited since clinical symptoms are nonspecific in children, they are observed at low rates and may be confused with many upper respiratory tract infections. COVID-19 infection can affect many laboratory parameters in children (3). In a study conducted in Italy, $36.8 \%$ and $\mathbf{1 5 . 7 \%}$ leukopenia and lymphopenia were detected among the patients, respectively, while AST and ALT increases were reported as $18.3 \%$ and $11.8 \%$, respectively (3). Henry et al., In their study conducted on 66 children, determined normal leukocyte counts $(69.2 \%)$, neutropenia $(6.0 \%)$, neutrophilia $(4.6 \%)$, lymphopenia (3.0\%), high C-reactive protein (13.6\%) and high procalcitonin (10.6\%). 20 children were evaluated in a study published in China, it was determined that leukopenia (20\%), lococytosis (10\%), lymphopenia (35\%), lymphocytosis (15\%), high ALT (25\%), high creatine kinase-MB as (75\%) (11). In this study, it was determined that high CRP (35.3\%), ALT (5.8\%), high AST (11.5\%), high WBC as (23.7\%) and low WBC as (14.4\%), while high level of ALT, AST, WBC, Lymphocyte were determined as statistically significantly higher in younger age group children and high neutrophilia in older age group. In the light of given data, it is possible to say that laboratory parameters are important parameters in the diagnosis and follow-up of COVID-19. Some recent studies have reported that CT imaging, especially in adults, may have a high sensitivity and prognostic value (5). However, since the severity and frequency of COVID-19 pneumonia is observed lower in pediatric patients in comparison with adults, the imaging findings, mode 
of involvement and the role of CT imaging may differ from adult patients $(6,7)$. In a study conducted in Italy, it was reported that $41.5 \%$ of children with chest X-ray had ground glass opacity and $9.8 \%$ had focal consolidation (3). In another study, it was reported that there was $29.33 \%$ no abnormality in CT imaging, $29.3 \%$ local patch shading, $34.6 \%$ bilateral patched shading, and $6.67 \%$ ground glass opacity (3). In another study, it was reported that there was $20 \%$ normal, $30 \%$ unilateral and $50 \%$ bilateral involvement on chest CT (11), in another, CT findings that reminded the infection were found in $25.9 \%$ of pediatric patients (19), in this last study, the presence of lung Rx findings (48.2\%) and CT findings (24.5\%) were detected in the patients. It is understood that lung CT imaging is a very limited test in diagnosis and follow-up, since COVID-19 infection in pediatric cases is generally less common and milder in comparison with adults. The standard test for the diagnosis of COVID-19 is considered as the RT-PCR test, especially in patients without obvious clinical findings (5). While PCR test positivity makes the diagnosis of COVID-19 with full accuracy, PCR test negativity may be seriously insufficient to determine the diagnosis of COVID-19. The major reason for this is the high false negativity of the PCR test due to many factors, especially in children and adult COVID-19 patients without obvious clinical findings (5). Specifically, in the largest study from China, most cases were diagnosed as an outpatient and only $34.1 \%$ of cases were confirmed by the laboratory (20). At the time of diagnosis, $13-15 \%$ of virologically positive children may be asymptomatic $(13,15)$. While in this study, the PCR positivity rate was determined as $43.9 \%$, the frequency of fever, high CRP, leukocytosis, high neutrophil and CT findings were detected statistically significantly higher in PCR (-) cases compared to PCR $(+)$ cases, frequency of respiratory distress, high lymphocyte and CT findings were significantly lower. Within the scope of given data, it is understood that when the PCR test is used for diagnostic purposes in children, it is a test with low sensitivity and it may cause false-negative, especially in cases with fever, high CRP, leukocytosis, high neutrophil and respiratory distress in CT findings.

As a result, COVID-19 infection may indicate different nonspecific clinical, laboratory and radiological findings in children not only compared to adults but also among pediatric age groups. In addition, PCR test results are being affected by conditions like fever, respiratory distress, high CRP, leukocytosis, high neutrophil and CT finding. We believe that further comprehensive studies are needed on this subject.

\section{Acknowledgment}

We confirm that no conflicts of interest for all Authors.

\section{REFERENCES}

1. Grasselli G, Pesenti A, Cecconi M. Critical Care Utilization for the COVID-19 Outbreak in Lombardy, Italy: Early Experience and Forecast During an Emergency Response. JAMA. 2020 Apr $28 ; 323(16): 1545-1546$

2. World Health organization. Coronavirus disease (COVID-2019) situation reports 79 . Available at (accessed on March 27, 2020) https://www.who.int/emergencies/diseases/novel-coronavirus2019/situation-reports

3. Peng H, Gao P, Xu Q, Liu M, et al. Coronavirus disease 2019 in children: Characteristics, antimicrobial treatment, and outcomes. J Clin Virol. 2020 Jul;128:104425.

4. Mohammadi A, Mohebbi I, Khademvatani K. et al. Clinical and radiological characteristics of pediatric patients with COVID-19: focus on imaging findings.Jpn J Radiol. 2020 Jun 13 : 1-6.

5. Fang Y, Zhang H, Xie J, et al. Sensitivity of Chest CT for COVID-19: Comparison to RT-PCR. Radiology. 2020;296(2):E115-E117.

6. Kanne JP, Yandow DR, Meyer CA. Pneumocystis jiroveci pneumonia: high-resolution CT findings in patients with and without HIV infection. Am J Roentgenol. 2012;198(6):W555-W56161.

7. Kim J, Lee IJ, Kim JH. CT findings of pulmonary tuberculosis and tuberculous pleurisy in diabetes mellitus patients. Diagn Interv Radiol. 2017;23(2):112-117. doi:10.5152/dir.2016.16157

8. World Health Organization. (2020). Laboratory testing for coronavirus disease 2019 (COVID19) in suspected human cases: interim guidance, 2 March 2020. World Health Organization. https://apps.who.int/iris/handle/10665/331329

9. Dong Y, Mo X, Hu Y, et al. Epidemiological characteristics of 2143 pediatric patients with 2019 
coronavirus disease in China. J Emerg Med. 2020;58(4):712-713.

10. Shekerdemian LS, Mahmood NR, Wolfe KK, et al. Characteristics and Outcomes of Children With Coronavirus Disease 2019 (COVID-19) Infection Admitted to US and Canadian Pediatric Intensive Care Units [published online ahead of print, 2020 May 11]. JAMA Pediatr. 2020;10.1001/jamapediatrics.2020.1948.

11. Xia W, Shao J, Guo Y, et al. Clinical and CT features in pediatric patients with COVID-19 infection: Different points from adults. Pediatr Pulmonol. 2020;55(5):1169-1174.

12. Sinha IP, Harwood R, Semple MG, et al. COVID-19 infection in children. Lancet Respir Med. 2020;8(5):446-447.

13. Xu Y, Li X, Zhu B, et al. Characteristics of pediatric SARS-CoV-2 infection and potential evidence for persistent fecal viral shedding. Nat Med. 2020;26(4):502-505. doi:10.1038/s41591-020-0817-4

14. Jiehao C, Jin X, Daojiong L, et al. A Case Series of Children With 2019 Novel Coronavirus Infection: Clinical and Epidemiological Features. Clin Infect Dis. 2020;71(6):1547-1551. doi:10.1093/cid/ciaa198

15. Lu X, Zhang L, Du H, et al. SARS-CoV-2 Infection in Children. N Engl J Med. 2020;382(17):16631665.

16. Zhang $\mathrm{C}, \mathrm{Gu}$ J, Chen Q, et al. Clinical and epidemiological characteristics of pediatric SARS-CoV-2 infections in China: A multicenter case series. PLoS Med. 2020;17(6):e1003130. Published 2020 Jun 16.

17. Cao Q, Chen YC, Chen CL, et al. SARS-CoV-2 infection in children: Transmission dynamics and clinical characteristics. J Formos Med Assoc. 2020;119(3):670-673.

18. Henry BM, Lippi G, Plebani M. Laboratory abnormalities in children with novel coronavirus disease 2019. Clin Chem Lab Med. 2020;58(7):1135-1138.

19. Richardson P, Griffin I, Tucker C, et al. Baricitinib as potential treatment for 2019-nCoV acute respiratory disease [published correction appears in Lancet. 2020 Jun 20;395(10241):1906].

20. Pedersen SF, Ho YC. SARS-CoV-2: a storm is raging. J Clin Invest. 2020;130(5):2202-2205.

\section{Hosted file}

Tables.pdf available at https://authorea.com/users/374597/articles/507367-evaluation-ofclinical-laboratory-and-radiological-findings-in-pediatric-covid-19-cases-and-their-

relation-with-rt-pcr-result 